\title{
Are There AGNs in the Nearby Dwarf Galaxies?
}

\author{
I. D. Karachentsev
}

Special Astrophysical Observatory, Russian Academy of Sciences, N.Arkhyz, 369167, Russia

\section{E. Karachentseva}

Astronomical Observatory of Kiev University, 04053, Observatorna 3, Kiev, Ukraine

\begin{abstract}
The presence or absence of a nucleus is considered for a sample of galaxies limited by distance, not by flux. For the 365 nearest galaxies situated within a distance of $\sim 7 \mathrm{Mpc}$, classification of their central regions is based on large-scale images obtained with the Hubble Space Telescope as well as with the 6-m and other large ground-based telescopes. Occurrence of nucleated galaxies is considered as a function of their luminosity, morphological type and other global properties.
\end{abstract}

\section{Introduction}

The problem of studying galactic nuclei during the time elapsed since the Byurakan symposium "Nonstationary Events in Galaxies" (1965) has come to be more sharply defined. The apearance of numerous new data are due to the improvement of observational facilities; and using these data, in turn, resulted in the development of theoretical insight into the processes that occur in the nuclei of galaxies. Note, however, that the subject of investigation itself is not infrequently defined either by mutual agreement as a certain central structure in a galaxy, a "nucleus", or - depending on the task stated, observational possibilities or the taste of authors - it breaks down into quite a few definitions. For illustration, let us present a few classification schemes with no pretence to their completeness.

Byurakan Classification (Kalloglian \& Tovmassian 1964; Tovmassian 1965; Tovmassian 1966; Sahakian 1968, and papers cited therein.) The galaxies here are divided into five classes: " 1 " - no nucleus (i.e. in the central parts of the galaxy there are no signs of any condensation); " 2 " - a faint nucleus is supposed to exist against the background of the central part; " 3 " - the nucleus does not stand out against the bright central condensation; " 4 " - - the nucleus is not completely star-like; "5" - the nucleus is practically star-like, the contrast to the central part is high.

Vorontsov-Velyaminov's Classification (Vorontsov-Velyaminov 1965). It divides the "nuclear regions of galaxies" into: disk " $\mathrm{D}$ " whose thickness is roughly equal to the thickness of the spiral arms. Found only in late spirals; lens " $\mathrm{"} \mathrm{-}$ more or less dense structure, with brightening towards the center. Encountered 
only in Sa and Sb galaxies; bulge "B" - somewhat different from the lens by greater sphericity and a more rapid drop of brightness from the center towards the edge. Characteristic of S0 and SBO galaxies; nucleus "N" - structure resembling a bulge, but relatively more compact. Characteristic of $\mathrm{Sb}, \mathrm{Sbc}, \mathrm{Sc}$; kernel " $K$ " - star-like condensation of very small size. They are found in some $\mathrm{E}$ galaxies and are likely to be absent in diffuse dwarf Sculptor-type galaxies in the Local group.

From the papers of the 1960s the classification according to Deitch (1966) can be mentioned. Papers by Kalloglian \& Tovmassian (1964); Tovmassian (1965); Tovmassian (1966); Sahakian (1968); Vorontsov-Velyaminov (1965); and Deitch (1966) were based on photographic data. In each series of classification, about 200-300 galaxies of different types were examined. The authors noted satisfactory agreement of the characteristics which describe the central regions of galaxies in different schemes. Note that these early papers did not seek to classify galaxies from a homogeneous (flux-limited or volume-limited) sample. Their object was the search for the regularities associated with the variation of the class/brightness of the nucleus depending on the morphological type/luminosity of the host galaxy.

Van den Bergh's Classification (van den Bergh 1995). Using the data of "The Atlas of Galaxies" (Sandage \& Bedke 1988), 342 central regions of late-type galaxies in the Shapley-Ames Catalog with $m_{p g} \leq 12^{m} 0$ (flux-limited sample) are classified here. The classification scheme: $\mathrm{NN}$ - no nucleus, $\mathrm{N}$ - star-like nucleus, SSN - semistellar nucleus, CB - a small central bulge or a disk is visible in the center of the image, NB - bar-like structure in the center, $\mathrm{Tr}$ - transient objects, intermediate between spirals, having a central bulge, and objects with the central regions resolved into stars and knots.

In the 1980s-1990s many new papers appeared which were concerned with CCD photometry of early-type galaxies (Binggeli \& Cameron 1991) in the Virgo and Fornax clusters, as well as individual galaxies (Kormendy 1985; Lauer et al. 1995). Without touching upon the interpretation of data, which is not infrequently fundamentally different, as in papers by Binggeli \& Cameron (1991) and Caldwell \& Bothun (1987), it will be noted that their authors point to the presence of either a rather dense and contrasty "bulge" in the central regions, or a diffuse "bulge", or a star-like "spike" superposed on the bulge (in the case of E galaxies), or on the body of the galaxy (in the case of dSph).

Detailed studies of $42 \mathrm{E}$ galaxies with the HST (Lauer et al. 1995) allow their nuclear regions to be classified by the character of the surface brightness distribution as "core", "power law" and "nuclear star clusters". We will return later to the question of existence of nuclei in dwarf spheroidal galaxies.

\section{The Local Volume Sample}

Before passing to the description of our sample, two basic remarks will be made. 1. Any statistical study of galaxies (in our case it is classification and determination of the frequency of occurrence of nuclei in galaxies of different types) must be performed from a volume-limited sample as complete as possible. With a diameter-limited or a magnitude-limited sample allowance should be made for different selection effects. 2. Dwarf galaxies of low luminosity and surface bright- 
ness are practically not represented in all general whole-sky catalogs. This refers also to the catalog of 179 nearby galaxies of Kraan-Korteweg and Tammann (1979) with $D \lesssim 10 \mathrm{Mpc}$, (KKT). The KKT sample has been complemented by Karachentsev (1994) from literature data and enhanced to $\mathrm{N}=216$ (galaxies of the Local Volume, $\mathrm{LV}$, with $V_{L G}<500 \mathrm{~km} / \mathrm{s}$ ). On the basis of using the sky surveys POSS-II and ESO/SERC, the authors started quests for dwarf galaxy candidates with an angular diameter $a \gtrsim 0.5$ in vicinities of the LV galaxies in order to make the sample still more complete (Karachentsev 1994). 260 galaxies (KK-galaxies) predominantly of low surface brightness have been found; half of them have been cataloged for the first time. $\mathrm{H}$ I observations of KK-objects with the 100-m radio telescope in Effelsberg (Huchtmeier et al. 1997; Huchtmeier et al. 2000) confirmed many of them as very nearby galaxies. By the present time, the whole-sky searches for dwarf candidates have been practically accomplished (Karachentseva et al. 1999) - KKR-objects; (Karachentseva \& Karachentsev 2000) - southern KKs-objects; (Karachentsev et al. 2000) - KKSG-objects; (Karachentsev et al. 2001) - KKH-objects). A total of over 600 candidates for the LV members were detected, about 300 of them for the first time.

Further observations added about 100 new dwarf galaxies to the LV. Together with the objects found by other researchers, the number of Local Volume galaxies, as compared to the KKT sample, has increased by more than a factor of two, and to-date $\mathrm{N}=365$. When preparing this new sample, we united and arranged in an ordered fashion our results obtained in papers (Karachentseva \& Karachentsev 1998), [18-21] (Karachentseva et al. 1999; Karachentseva \& Karachentsev 2000; Karachentsev et al. 2000; Karachentsev et al. 2001) as well as new data from the literature. As a result, we now have the following characteristics of the LV galaxies: the galaxy name, the equatorial coordinates, apparent total magnitude, radial velocity reduced to the Local group according to (Karachentsev \& Makarov 1996), morphological type of the galaxy in the digital code (so, $\mathrm{T}=10$ corresponds to irregular dwarfs, while $\mathrm{N}=-5$ to the faintest dwarfs dSph), distance of the galaxy in Mpc, luminosity logarithm in units $L_{\odot}$. We will comment in detail on our classification of the nuclear region of the galaxy in the next section.

\section{Observational Data and Classification Scheme}

As our experience shows, the photographic sky surveys POSS-II, ESO/SERC and SERC EJ on the whole serve for the morphological classification of nearby galaxies. However, the classification of their nuclei requires other data because the galaxy central regions usually appear to be over-exposed and dusty.

We have collected and analyzed the images accesible to us of LV galaxies from different sources (we enumerate them in the order of preference): 2MASS Catalog (Jarrett et al. 2000), the archives of the HST data, CCD images obtained with the 6-m and Nordic telescopes, the Atlas of Galaxies (Sandage \& Bedke 1988), POSS-II, ESO/SERC and SERC EJ sky surveys. We have also used photographs of LV galaxies from different publications. We succeeded in doing classification of many objects from our list independently for several sources. 
For the problem stated it turned out to be sufficient to isolate three main types of nuclear regions:

- "K" (kernel) — star-like "nucleus"

- "B" (bulge) - rather dense, but extended "nucleus"

- "A" - no "nucleus".

The following types in other classification schemes correspond approximately to these main types (Table 1 ):

Table 1. A correspondence between the KK and other authors galaxy

nuclei classification scheme

\begin{tabular}{cccc}
\hline This work & Byurakan & Vorontsov-Velyaminov & van den Bergh \\
\hline $\mathrm{K}$ & 5 & $\mathrm{~N}, \mathrm{~K}$ & $\mathrm{~N}$ \\
$\mathrm{~B}$ & $2:, 3,4:$ & $\mathrm{L}, \mathrm{B}, \mathrm{N}:$ & $\mathrm{CD}, \mathrm{NB}, \mathrm{SSN}:$ \\
$\mathrm{A}$ & $1,2:$ & - & $\mathrm{NN}$ \\
\hline coincidence, & $7 / 11$ & $6 / 9$ & $31 / 35$ \\
$\%$ & $63 \%$ & $67 \%$ & $89 \%$ \\
\hline
\end{tabular}

Thus, the concepts "star-like nucleus" or "no nucleus" in the schemes considered are described practically by one symbol. If a nuclear region is seen as extended, then several designations of other authors correspond to our symbol "B".

The results of the comparison are quite explicable: for classifying central regions of galaxies, large-scale images are most suitable. The inclusion of the 2MASS survey, where the influence of dust is practically removed, provides additional advantages for classification. Figure 1 gives some examples of classification of nuclear regions.

\section{Results and Conclusions}

Fig.2 presents the distribution of the number of galaxies in the LV with different types of central regions versus the distance of the galaxy, D, Mpc. As one can see, the median values of $D$ for "K", "B" and "A" objects are about the same, i.e., no strong selection with distance. At median $\mathrm{D}=5 \mathrm{Mpc}\left((m-M)_{0}=28.5\right)$ the detection limit of a nucleus $m_{\text {lim }} \simeq 18^{\mathrm{m}} 5$ corresponds to $M \simeq-10^{m}$. The angular resolution of the 2MASS survey at the same distance is approximately equal to $40 \mathrm{pc}$, and $\simeq 2 \mathrm{pc}$ corresponds to the angular resolution of the HST, $\simeq 0^{\prime \prime} 1$.

The occurrence of different nuclear types per galaxy in in the Local Volume is $f_{K}=23 / 365=6 \%$ for galaxies with kernels, and $f_{B}=33 / 365=9 \%$ for galaxies with bulges. The Local Volume density of nuclei is $V_{K}=0.014 / \mathrm{Mpc}^{3}$, and $V_{B}=0.023 / \mathrm{Mpc}^{3}$ within $D \sim 7 \mathrm{Mpc}$.

The distribution of the number of galaxies "K", "B" and "A" vs. host galaxy morphological type is displayed in Fig.3. Galaxies with bulges occur among all the types, kernels are observed in a few $\mathrm{E}$ galaxies and in late spirals. $\mathbf{K}$-type nuclei are absent in irregular and spheroidal dwarfs.

The distribution of the number of galaxies with different type nuclei vs. galaxy luminosity is shown in Fig.4. Galaxies with " $\mathbf{K}$ " and " $\mathbf{B}$ " have approxi- 

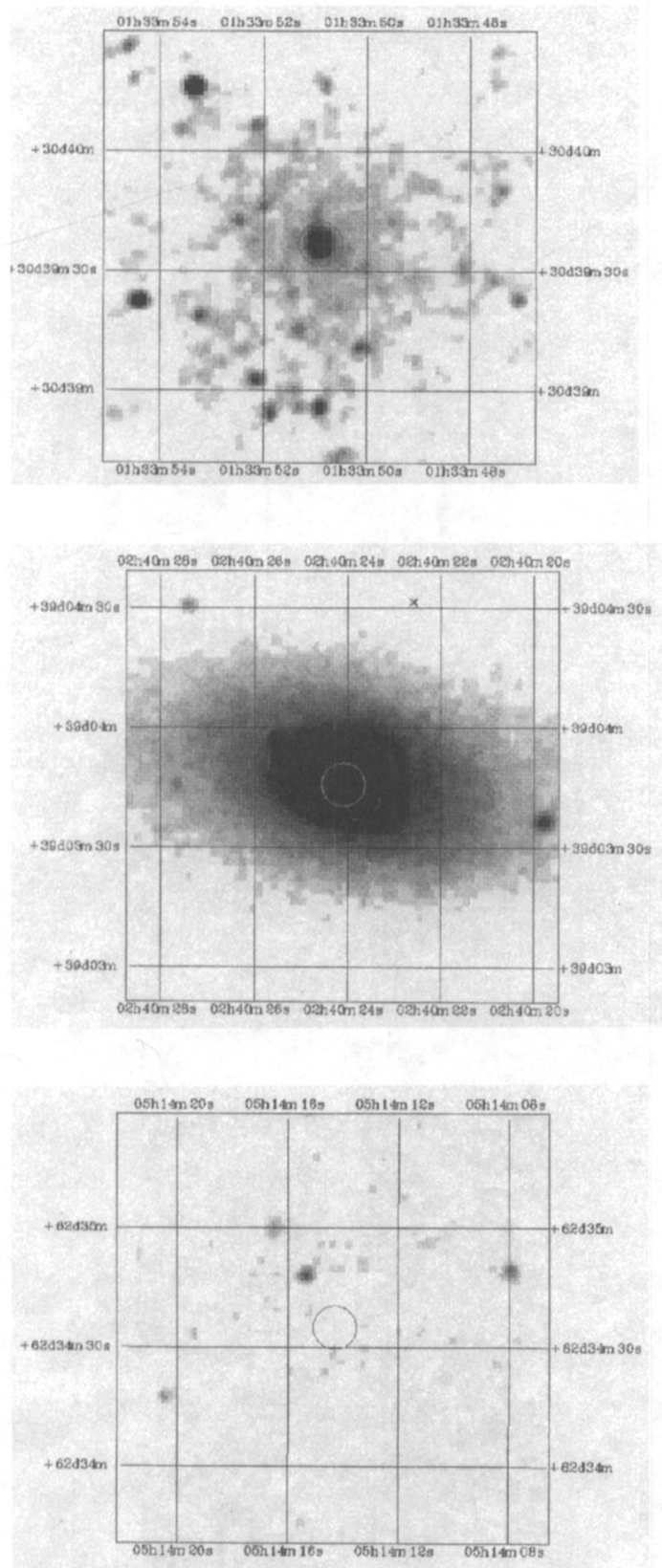

Figure 1. Examples of classification of nuclear regions (top: M33 K, middle: NGC 1023 - B, bottom: UGCA 105 - A). 


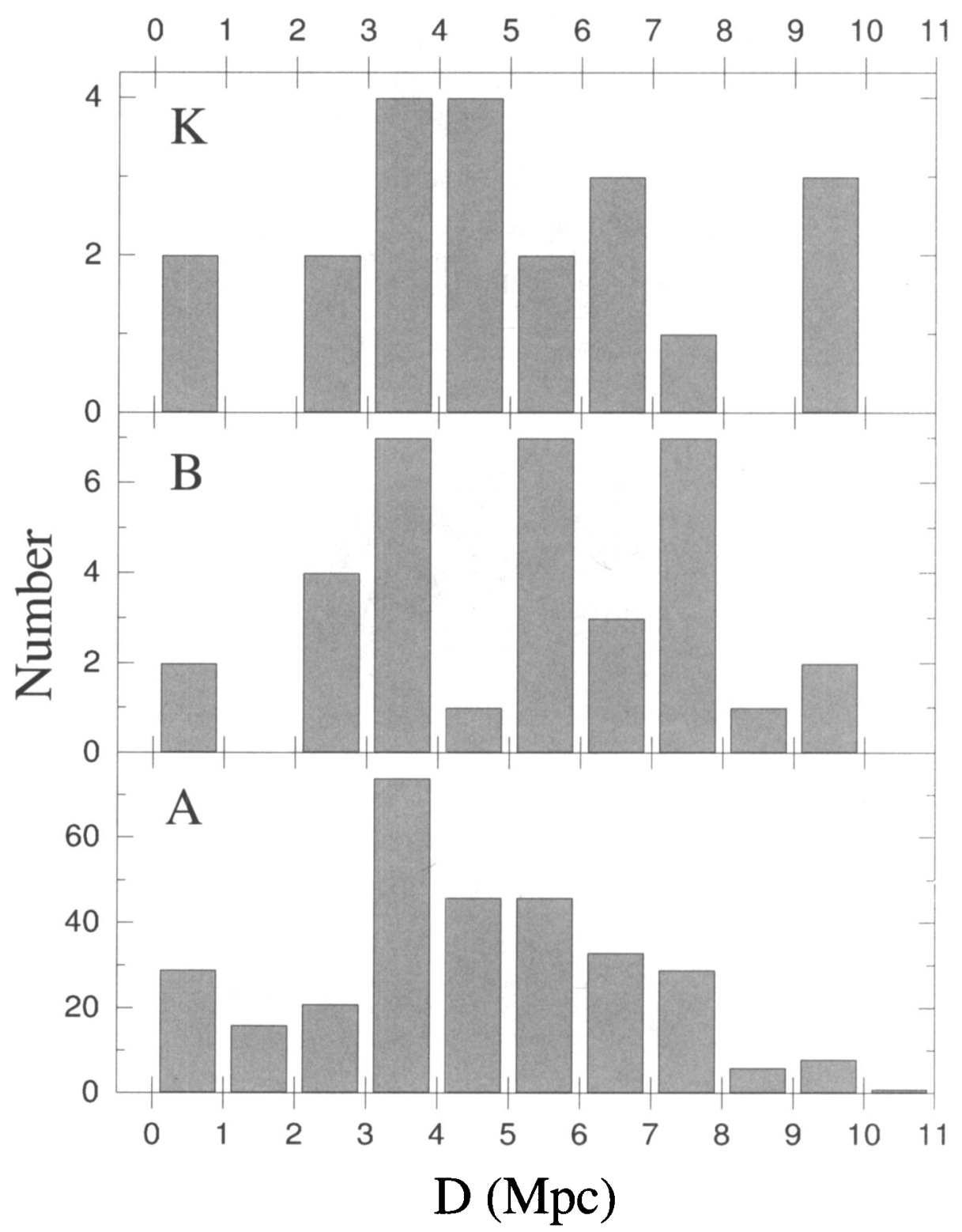

Figure 2. The distribution of the number of the LV galaxies with different nuclear region types versus the distance of the galaxy. 


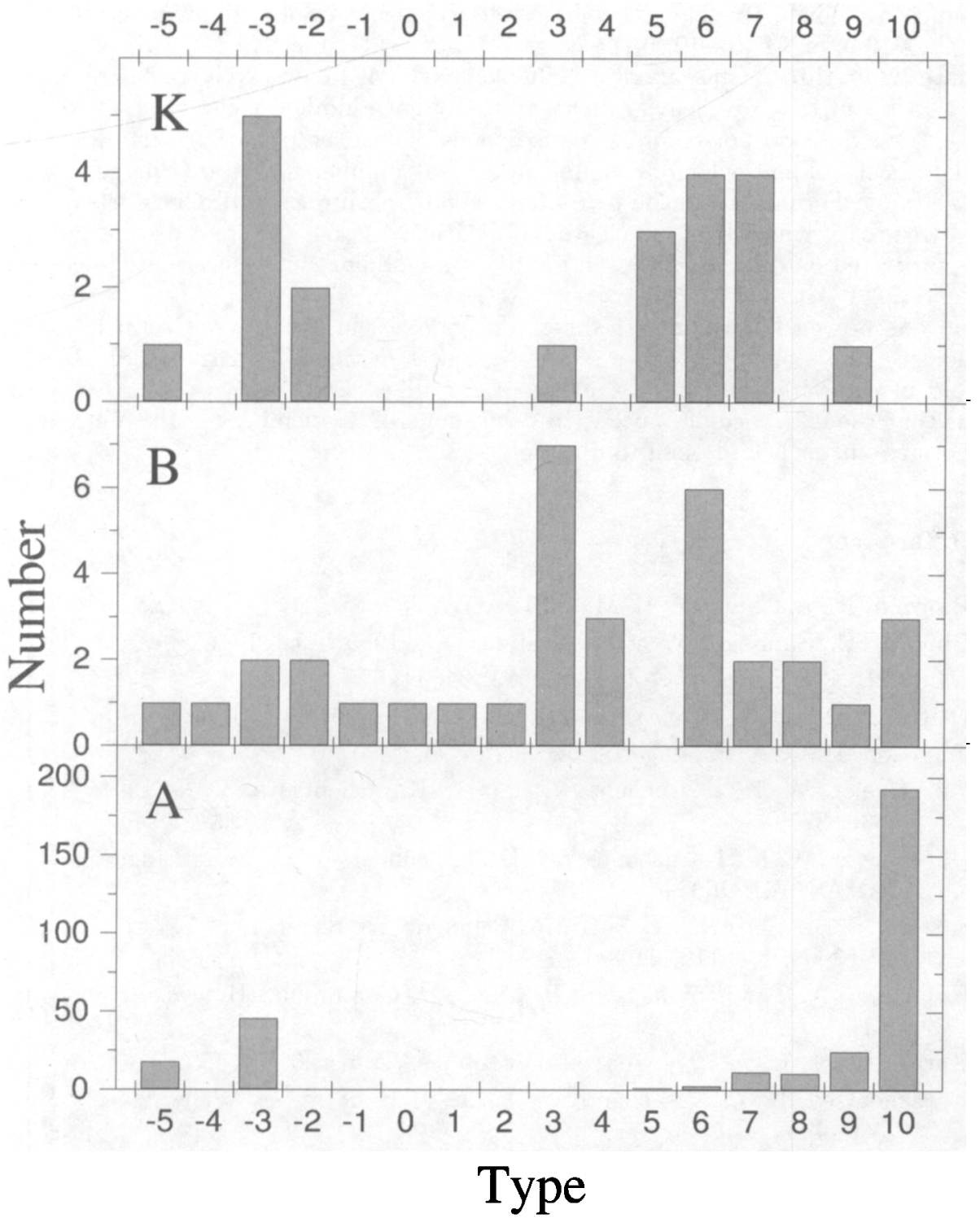

Figure 3. The distribution of the number of galaxies " $K$ ", "B", and "A" versus host galaxy morphological type. 
mately the same distributions in luminosity shifted by $5^{m}$ with respect to "A" galaxies. There are no " $\mathbf{K}$ " and "B" among dwarf galaxies (with an absolute magnitude $M>-15^{\mathrm{m}} 0$ ). A few exceptions are dwarfs brighter than $-15^{m}$, which are likely to belong to the Virgo cluster. Among giant galaxies with $l g L>10.0$, or $M<-19^{m} 6$ the cases "B" $(\mathrm{N}=14)$ and "K" $(\mathrm{N}=5)$ predominate. Only three giant galaxies: NGC 253 , NGC 4945 , and NGC 5236 are noted as "A", but they are heavily dusty and may have hidden nuclei.

Our data do not confirm the existence of "nucleated" dSph/dE dwarfs in the Local Volume, which are found in a great number in Virgo (Binggeli et al. 1985 ) and Fornax (Ferguson \& Sandage 1988). Examples of dSph dwarfs in the M 81 group, which look "nucleated", are BK5N, K 64 - here a distant galaxy is projected onto their center, and K 61 - a globular cluster is situated near its center.

As is well known, dwarf spheroidal galaxies in the Local Group have no nuclei. The global properties of these objects in the LG, in the M 81 Group, and in the Virgo and Fornax Clusters are alike. For this reason, it seems to us that the existence of "nuclei" in dwarf spheroidal members of the Virgo and Fornax cluster is still open to question.

\section{References}

Binggeli, B., \& Cameron, L. M. 1991, A\&A, 252, 27

Binggeli, B., Sandage, A., \& Tammann, G. A. 1985, AJ, 90, 1681

Caldwell, N. \& Bothun, G. D. 1987, AJ, 94, 1126

Deitch, A. N. 1966, Publ. Main Astron.obs.(Pulkovo), N.179, 95

Ferguson, H. C., \& Sandage, A. 1988, AJ, 96, 1520

Huchtmeier, W. K., Karachentsev, I. D. \& Karachentseva, V. E. 1997, A\&A, 332,375

Huchtmeier, W. K., Karachentsev, I. D., Karachentseva, V. E. \& Ehle, M. 2000, A\&AS, 141, 469

Jarrett, T. H., Chester, T., Cutri, R., Schneider, S. Skrutskie, M., \& Huchra, J. P., 2000, AJ, 119, 2498

Kalloglian, A. T. \& Tovmassian, H. M., 1964, Communic. Byurakan. obs., 36, 31

Karachentsev, I. D., 1994, Astron. Astrophys. Trans., 6, 1

Karachentsev, I. D., Karachentseva, V. E., \& Huchtmeier, W. K. 2001, A\&A, 366,428

Karachentsev, I. D., Karachentseva, V. E., Suchkov, A. A., \& Grebel, E. K. 2000, A\&AS, 145, 415

Karachentsev, I. D. \& Makarov, D. I., 1996, AJ, 111, 794

Karachentseva, V. E., \& Karachentsev, I. D. 1998, A\&AS, 127, 409

Karachentseva, V. E., Karachentsev, I. D. \& Richter, G. M. 1999, A\&AS, 135, 221

Karachentseva, V. E., \& Karachentsev, I. D. 2000, A\&AS, 146, 359

Kormendy, J. 1985, ApJ, 295, 73 


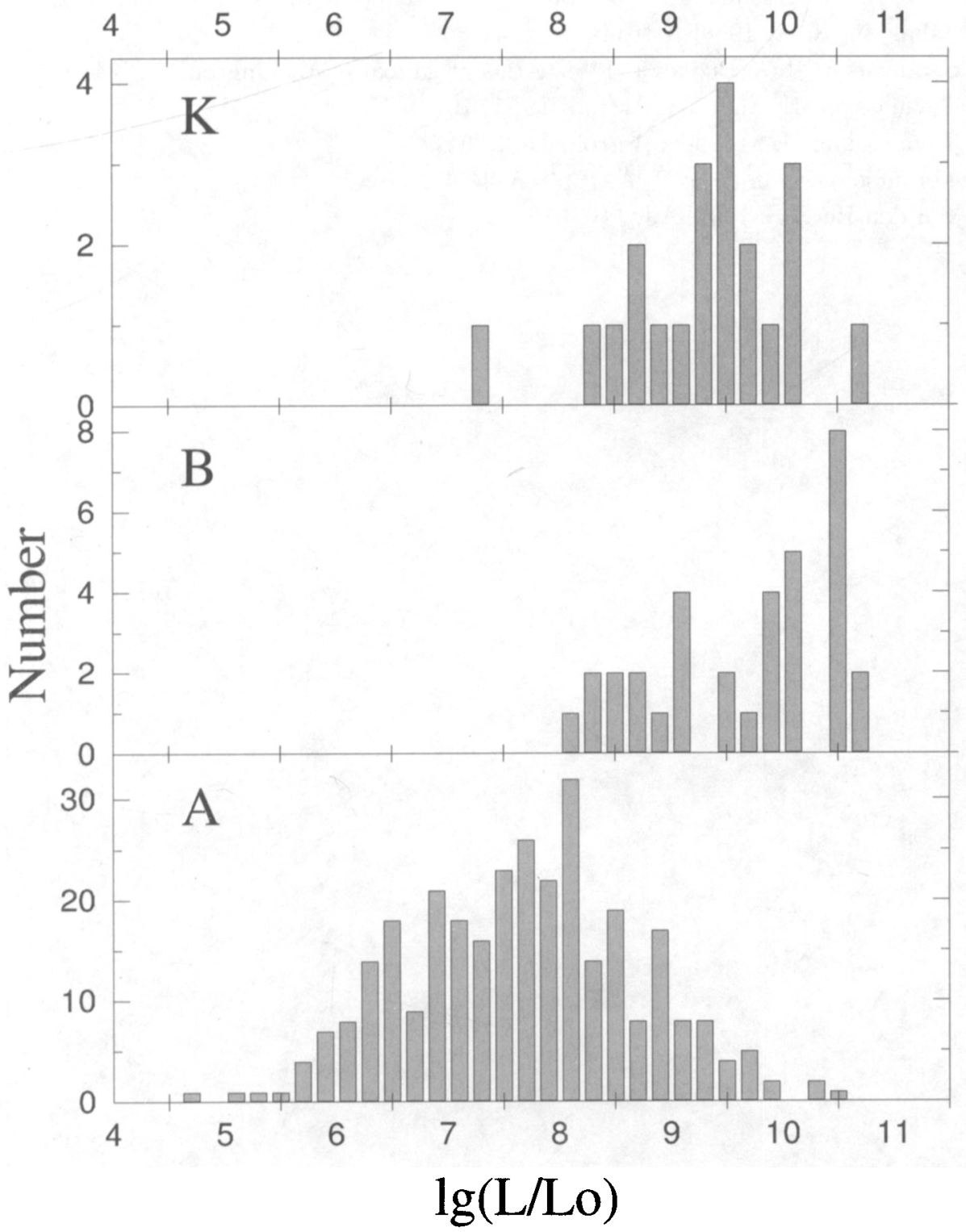

Figure 4. The distribution of the number of galaxies with different nuclear region types versus galaxy luminosity. 
Kraan-Korteweg, R. C., \& Tammann, G. A. 1979, AN, 300, 181

Lauer, T. R., Ajhar, E. A., Byun, Y. I. K., Dressler, A, Faber, S. M., Grilimair,

C., Kormendy, J., Richstone, D., \& Tremaine, S. 1995, AJ, 110, 2622

Sahakjan, K. A. 1968, Astrofizika, 4, 41

Sandage, A. R. \& Bedke, J. 1988, Atlas of galaxies, Washington, DC

Tovmassian, H. M. 1965, Astrofizika, 1, 197

Tovmassian, H. M. 1966, Astrofizika, 2, 317

Vorontsov-Velyaminov, B. A. 1965, AZh, 42, 1168

van den Berg, S. 1995, AJ, 110, 613 